Relations industrielles

Industrial Relations

\title{
Accroissement de la productivité et psychologie du travail, Bruxelles, Éditions de l'Université de Bruxelles, 1976, 122 pp.
}

Alain Vinet

Volume 34, numéro 1, 1979

URI : https://id.erudit.org/iderudit/028948ar

DOI : https://doi.org/10.7202/028948ar

Aller au sommaire du numéro

Éditeur(s)

Département des relations industrielles de l'Université Laval

\section{ISSN}

0034-379X (imprimé)

1703-8138 (numérique)

Découvrir la revue

Citer ce compte rendu

Vinet, A. (1979). Compte rendu de [Accroissement de la productivité et psychologie du travail, Bruxelles, Éditions de l'Université de Bruxelles, 1976, 122 pp.] Relations industrielles / Industrial Relations, 34(1), 203-204.

https://doi.org/10.7202/028948ar

Tous droits réservés @ Département des relations industrielles de l'Université Laval, 1979
Ce document est protégé par la loi sur le droit d'auteur. L'utilisation des services d’Érudit (y compris la reproduction) est assujettie à sa politique d'utilisation que vous pouvez consulter en ligne.

https://apropos.erudit.org/fr/usagers/politique-dutilisation/ 
Accroissement de la productivité et psychologie du travail, Bruxelles, Éditions de l'Université de Bruxelles, 1976, $122 \mathrm{pp}$.

Cet ouvrage collectif regroupe six études présentées par des chercheurs du Centre de sociologie du travail de l'Université de Bruxelles et financées par l'Office belge pour l'accroissement de la productivité (O.B.A.P.). La question de la productivité constitue évidemment le lien qui unit ces études par ailleurs fort diverses quant aux méthodes utilisées, aux entreprises et aux problèmes étudiés. Parmi ces derniers, le mode de rémunération et la gestion sociotechnique des entreprises sont davantage approfondis. La diversité des approches privilégiées par les auteurs incite à présenter séparément chacune de ces études.

KARNAS, Arielle, "Travail en groupe et mode de rémunération lié aux résultats". Cette étude vise à dégager le rôle du mode de rémunération sur les attitudes et les comportements d'un groupe de travail. Le sujet n'est pas nouveau mais l'angle d'approche et certaines conclusions de l'auteur méritent d'être soulignés. Les groupes de travail étudiés provenaient du secteur de la distribution (vente d'ameublement, de tapis, d'appareils électro-ménagers). Les entreprises en cause utilisaient trois modes de rémunération: rémunération fixe, rémunération liée aux résultats individuels et rémunération liée aux résultats collectifs. Considérant qu'il s'agit d'un milieu de travail extrêmement compétitif, l'auteur s'attache aux différentes stratégies utilisées par les vendeurs selon qu'ils sont soumis à l'un ou l'autre des modes de rémunération: " $s$ 'arracher des ventes " lorsque la rémunération est liée aux résultats individuels, "tirer au flanc» dans les deux autres cas. Mais l'intérêt de cet article tient plutôt à une conclusion formulée par Karnas à la suite de l'observation des mécanismes de surveillance réciproque mis au point par les vendeurs: le groupe de travail, quel que soit le mode de rémunération, est perçu par ses membres comme un facteur important d'aliénation, une entrave sérieuse à l'autonomie. On aurait souhaité que l'auteur approfondisse cette question et qu'elle discute davantage des fonctions régulatrices du groupe de travail.
PONCIN, Anny, «Motivations, attitudes et systèmes d'organisation du travail ». Cet article s'attarde particulièrement aux liens entre les modes de rémunération et l'organisation du travail. L'auteur y poursuit une hypothèse qui n'est pas nouvelle mais qui la guide vers des constatations intéressantes: désireuse de montrer que dans le secteur de la distribution, les modes de rémunération sont associés à l'évolution des techniques de vente, Poncin est amenée à s'intéresser aux conséquences de cette évolution sur les motivations et les attitudes du personnel de vente. Elle constate que l'apparition et la généralisation de techniques de ventes caractérisées par le «libre service " provoquent l'éclatement des tâches, des vendeurs: "les tâches d'encaissement et de manutention sont dissociées, la fonction de vente dans son acceptation large d'argumentation et d'information s'efface peu à peu». À ces fonctions de plus en plus simplifiées correspond un retour à une rémunération fixe. L'auteur conclut qu'on assiste présentement à une déqualification des travailleurs dans le secteur de la distribution comme dans d'autres secteurs et que cette déqualification est vivement ressentie par le personnel de vente.

LACOMBLEZ, Marianne, «Flexibilité professionnelle et perception instrumentale du travail». Cette étude de caractère monographique est le résultat de l'insertion d'un chercheur dans une usine de montage automobile nouvellement implantée dans une région désignée. L'étude fut motivée par le haut niveau d'absentéisme constaté dans l'entreprise et par un taux de roulement des employés voisinant $65 \%$. Or l'arrivée de l'entreprise dans la région était manifestement attendue avec beaucoup d'enthousiasme par la main-d'œuvre locale. Comment expliquer dans ces conditions le comportement des ouvriers? L'auteur constate que l'usine de montage automobile fut implantée dans un milieu doté d'une tradition et d'une culture ouvrières incitant l'individu à considérer le travail comme un moyen de réalisation de soi. Ces ouvriers «traditionnels» n'étaient aucunement préparés aux conditions de travail prévalant dans l'usine: éclatement des tâches et perception instrumentale du travail. La désillution de la main-d'œuvre régionale serait à l'origine des hauts niveaux d'absentéisme 
et de roulement. Lacomblez conclut que la flexibilité de la main-d'œuvre n'est pas aussi grande qu'on le croit généralement et que seule une approche socio-technique incluant «la prise en considération du monde extra-entreprise" (la région, son passé, son histoire) est susceptible de favoriser une adaptation réciproque de l'entreprise et de la main-d'œuvre régionale.

C'est la démarche que propose Eric VERBORGH dans une étude de caractère plus théorique intitulée «Organisation du travail et gestion socio-technique». Cet article décrit un cadre conceptuel et méthodologique connu, visant à considérer l'entreprise comme un système sociotechnique ouvert, en relation permanente avec son environnement. L'influence des facteurs externes sur le système social de l'entreprise y est particulièrement discutée ainsi que la primauté générale du système technique sur le système social. L'auteur offre un modèle explicatif des diverses influences s'exerçant sur et au sein de l'entreprise.

Enfin, deux articles plus brefs complètent cette sélection. Dans sa présentation intitulée "Relations entre services et intervention dans l'entreprise», Jean Pierre VANDENBOSH décrit quelques-unes des difficultés rencontrées par le psychologue lors de ses interventions auprès de l'entreprise. Pour sa part, Marcel BOLLE DE BAL discute brièvement des problèmes posés par l'apparition de méthodes perfectionnées de qualification du travail (job evaluation) et s'interroge sur l'avenir de celles-ci.

Bref, cet ouvrage présente à l'occasion des points de vue originaux sur des sujets connus ou déjà fouillés. Il ne s'agit pas cependant (les auteurs ne le prétendent pas non plus) d'un document de pointe ou d'un bilan des connaissances accumulées dans ce secteur.

Alain VINET

Université Laval

Organized Behavior: an Applied Psychological Approach, by Clay Hamner et Dennis W. Organ, Dallas, Texas, Business Publications Inc., 1978, 437 pp.
La majorité des programmes d'enseignement en administration, en psychologie, en éducation, en relations industrielles offrent sous une forme ou une autre des cours et des séminaires qui traitent du comportement des individus et des groupes au sein des organisations de travail. La diversité de ces cours et séminaires tant au plan du contenu que de la pédagogie utillisée soulève bien des questions quant à la nature même de ce champ de connaissances qu'est le comportement organisationnel. Existe-t-il, en effet, un noyau (core) de connaissances assez cohérent et assez homogène pour constituer une trame commune à tous ces enseignements? Les auteurs de cet ouvrage prétendent qu'à la lumière des développements récents de la psychologie et de la sociologie des organisations, il y a lieu de constater que le «comportement organisationnel» est beaucoup plus qu'un champ de connaissances et qu'il est en train d'accéder au statut de discipline académique avec un objet qui lui est propre, un mode de raisonnement et d'observation contrôlée adaptée des méthodes de recherches en sciences sociales. Leur prétention semble suffisamment fondée puisqu'ils ont réussi à identifier les thèmes qui constituent l'objet propre de ce domaine et qu'on retrouve de façon recurrente dans la diversité des enseignements. Les trois grands thèmes qu'ils ont retenus sont les suivants: le comportement individuel, le comportement des groupes et les effets de ces comportements sur l'efficacité et la «santé » d'une organisation de travail dans un contexte socio culturel donné. Chacun de ces grands thèmes sont traités selon un cheminement logique et historique du développement des connaissances, cheminement qui est susceptible de plaire à l'intelligence du lecteur. Les processus d'apprentissage et leur application en matière de formation et d'adrninistration de la discipline, les perceptions, les attitudes, la motivation, les différences individuelles, le stress, la satisfaction au travail, les programmes sociaux récents d'amélioration de la qualité de la vie au travail sont autant de sujets traités sur la rubrique «comportement individuel».

La structure et le fonctionnement du groupe de travail, les phénomènes des conflits, de pouvoir, de leadership et du contrôle constituent le deuxième grand thème 